\title{
Impact of time since last caloric intake on blood glucose levels
}

\author{
Susanne Moebus • Laura Göres • Christian Lösch • \\ Karl-Heinz Jöckel
}

Received: 31 December 2010/ Accepted: 12 July 2011/Published online: 6 August 2011

(C) The Author(s) 2011. This article is published with open access at Springerlink.com

\begin{abstract}
Blood glucose (BG) is usually measured after a caloric restriction of at least $8 \mathrm{~h}$; however evidence-based recommendations for the duration of a fasting status are missing. Here we analyze the effect of fasting duration on levels of $\mathrm{BG}$ to determine the minimal fasting duration to achieve comparable BG levels to conventional fasting measurements. We used data of a cross-sectional study on primary care patients, performed in October 2005. We included 28,024 individuals (age-range 18-99 years; 63\% women) without known diabetes mellitus and without missing data for BG and fasting status. We computed general linear models, adjusting for age, sex, time of blood withdrawal, systolic blood pressure, waist circumference, total- and HDL-cholesterol, physical activity, smoking, intake of beta-blocker and alcohol. We tested the intraindividual variability with respect to fasting status. Overall, the mean BG differed only slightly between individuals fasting $\geq 8 \mathrm{~h}$ and those fasting $<8 \mathrm{~h}$ (men: $5.1 \pm 0.8 \mathrm{mmol} /$ $\mathrm{L}$ versus $5.2 \pm 1.2 \mathrm{mmol} / \mathrm{L}$; women: $4.9 \pm 0.7 \mathrm{mmol} / \mathrm{L}$, $5.0 \pm 1.0 \mathrm{mmol} / \mathrm{L}$ ). After $3 \mathrm{~h}$ of fasting differences of BG diminished in men to $-0.08 \mathrm{mmol} / \mathrm{L}(95 \%-\mathrm{CI}:-0.15$; $-0.01 \mathrm{mmol} / \mathrm{L})$, in women to $-0.07 \mathrm{mmol} / \mathrm{L}(-0.12$; $-0.03 \mathrm{mmol} / \mathrm{L}$ ) compared to individuals fasting $\geq 8 \mathrm{~h}$. Noteworthy, age, time of day of blood withdrawal, physical activity, and intake of hard liquor influenced BG levels
\end{abstract}

Electronic supplementary material The online version of this article (doi:10.1007/s10654-011-9608-z) contains supplementary material, which is available to authorized users.

S. Moebus $(\bowtie) \cdot$ L. Göres · C. Lösch · K.-H. Jöckel Institute for Medical Informatics, Biometry and Epidemiology, University Hospital of Essen, University of Duisburg-Essen, Hufelandstr. 55, 45122 Essen, Germany

e-mail: susanne.moebus@uk-essen.de considerably. Our data challenge the necessity for a fasting duration of $\geq 8 \mathrm{~h}$ when measuring blood glucose, suggesting a random sampling or a fasting duration of $3 \mathrm{~h}$ as sufficient. Rather, our study indicates that essentially more effort on the assessment of additional external/internal factors on BG levels is necessary.

Keywords Blood glucose - Risk assessment ·

Nonfasting $\cdot$ Cross-sectional study

$\begin{array}{ll}\text { Abbreviations } \\ \text { ADA } & \text { American Diabetes Association } \\ \text { BG } & \text { Blood glucose } \\ \text { CVD } & \text { Cardiovascular disease } \\ \text { GEMCAS } & \begin{array}{l}\text { GErman Metabolic and Cardiovascular riSk } \\ \text { Project }\end{array} \\ \text { HDL-C } & \text { High-density lipoprotein cholesterol } \\ \text { LDL-C } & \text { Low-density lipoprotein cholesterol } \\ \text { oGTT } & \text { Oral glucose tolerance test } \\ \text { T-C } & \text { Total cholesterol } \\ \text { TG } & \text { Triacylglycerol }\end{array}$

\section{Introduction}

The measurement of blood glucose is a well established procedure routinely used for many clinical and research purposes. In epidemiological studies blood glucose is an often measured parameter be it as a risk factor, mediator or confounder. Measuring blood glucose requires standardized procedures to minimize variability and bias, both in terms of required analytical methods and biological variability. Blood glucose levels are influenced by external factors, like caloric intake resulting in an increase of blood 
glucose or metabolic demands like muscle activity resulting in a decline of blood glucose. In an attempt to obtain unbiased blood glucose measurements one of the routinely requested basic requirements for pre-analytical blood sampling is the fasting state. However the fasting state is not well defined, i.e. the WHO recommends an 8-14 h (h) fast [1], the American Diabetes Association (ADA) defines fasting as "no caloric intake for at least $8 \mathrm{~h}$ " [2] or "an overnight 8- to 10-h fast" [3]. Moreover, evidence-based recommendations for the definition of the duration of the fasting status are missing-perhaps one reason, why blood glucose measurements in epidemiological and clinical studies are carried out inconsistently with regard to fasting duration. Pre-analytical blood sampling schemes range from overnight fast, fasting duration between $8 \mathrm{~h}$ and $>12 \mathrm{~h}, \geq 12 \mathrm{~h}$, random sampling to even no information at all.

In the clinical as well as in the research environment, the required fasting status - however defined-is a challenging task. For clinicians and patients it would be much simpler if a blood sample could be taken at any time of the day, irrespective of the fasting duration. In studies, especially epidemiological studies, fasting requirement influences the study design, complicates the field work and increases the costs of the study. Moreover, and most important, it is not feasible to reliably control for the self-reported fasting status.

So far, few studies have examined the association between fasting duration and blood glucose comprehensively. Most of them studied only the dichotomous approach of fasting versus non-fasting, using various cutpoints and not taking into account the course of blood glucose fluctuation according to duration of fasting status.

The aim of our study is to analyze the effect of fasting duration on levels of blood glucose to determine the minimal fasting duration to achieve comparable blood glucose levels to $>8 \mathrm{~h}$ fasting measurements. For this purpose we use the dataset of GEMCAS (GErman Metabolic and Cardiovascular riSk Project), a recent nationwide study.

\section{Methods}

Study design and study participants

GEMCAS was conducted during 2 weeks in October 2005 at 1,511 randomly selected primary care physicians across Germany. Methods have been previously described in detail $[4,5]$. Briefly, we included all eligible individuals aged 18-99 years visiting a general practitioner during these 2 weeks regardless of the reason of their visit and regardless of their fasting status. The study was planned and conducted according to the German guidelines for
Good Epidemiology Practices (GEP) [6]. All participants gave their written informed consent and the study protocol was approved by the institutional ethics committee.

\section{Data assessment}

Data were collected on sociodemographic variables, antihypertensive, lipid-lowering and diabetic medication, smoking habits, physical activity and time of day of blood withdrawal. Self-reported physician diagnosed history of CVD and diabetes mellitus was filled in by the physician. Type and time of the last caloric intake (meals and drinks) were documented, waist circumference and blood pressure measured according to the study protocol. For blood sampling a two-step approach was performed [5]. First, all participants underwent a screening by using a blood glucose quick test from a capillary (finger stick) sample. Accordingly, it was possible to directly exclude or diagnose hyperglycaemia in those participants with a fasting duration of $\geq 8 \mathrm{~h}$ and a capillary blood glucose of $<5.56$ or $>11.11 \mathrm{mmol} / \mathrm{L}$. If the findings concerning fasting serum glucose were ambiguous $(\geq 5.56$ or $\leq 11.11 \mathrm{mmol} / \mathrm{L})$ due to a meal in the previous $8 \mathrm{~h}$, the participant was asked to come for a re-examination to give a fasting blood sample. Furthermore, a random sample (30\%) of participants was asked to come for a second fasting blood sample, regardless of the result of the blood glucose quick test.

\section{Laboratory analysis}

For the main analysis a venous blood sample was collected from each participant, and shipped within $24 \mathrm{~h}$ to a central laboratory (Berlin, Germany) by an assigned courier service. Standardised enzymatic methods were used to determine total cholesterol (T-C), high-density lipoprotein cholesterol (HDL-C), low-density lipoprotein cholesterol (LDL-C), and triacylglycerol (TG) using Roche Hitachi MODULAR systems. Glucose levels from blood in natriumfluorid $(\mathrm{NaF})$-tubes were estimated by the glucose-6phosphate dehydrogenase method (G6P-DH).

Quality assurance

We implemented a comprehensive quality assurance, performing a special monitoring concept, which included telephone-monitoring and random on-site visits, recently described in detail [5].

Diagnostic conventions and covariates

The fasting state was defined by a fasting duration of at least $8 \mathrm{~h}$. Smoking was defined as current smoking or as a 
history of cigarette smoking during the past year. Physical inactivity was set by $<2 \mathrm{~h} /$ week. The time since last caloric intake was calculated by generating the difference between the time of blood withdrawal and time of last caloric intake (meal or drink) whereas missing data for one of the two variables was excluded.

\section{Statistical analyses}

Of the 35,869 study participants we excluded for the present analysis those with missing data for blood glucose ( $\mathrm{n}=1,236)$ and fasting status $(\mathrm{n}=1,947)$ as well as those with a fasting status $>35 \mathrm{~h}(\mathrm{n}=12)$. Since the impact of time of last caloric intake on blood glucose differed markedly between participants with and without known diabetes mellitus, we further excluded 4,650 participants with known diabetes mellitus, leaving a study sample of 28,024 (mean age $49.9 \pm 15.9$ years, $63 \%$ women). For analyses, where the exact time since last caloric intake was necessary, additional 2,489 participants were excluded $(n=25,535$; mean age $49.5 \pm 15.9$ years, $62 \%$ women).

Differences in levels of glucose as a function of time since last caloric intake were tested with the Student $t$ test between fasting levels $(\geq 8 \mathrm{~h})$ and the remaining 8 time points $\left(t_{0}, \ldots\right.$, $\mathrm{t}_{7}$ ). Tests were corrected for multiple comparisons with the Bonferroni method. To investigate the relationship between duration of fasting and age on blood glucose in more detail, we conducted analysis of covariance. We used continuous blood glucose as the dependent variable, and time since last caloric intake as categorical covariate $\left(\mathrm{t}_{1 \mathrm{~h}}, \ldots, \mathrm{t}_{\geq 8 \mathrm{~h}}\right.$ as reference) adjusting for age. To account for possible influences of other covariates on blood glucose we used general linear models including the variables age, sex, time of day of blood withdrawal (dichotomized as morning, <12.00 a.m., and afternoon), systolic blood pressure, waist circumference, HDL-cholesterol, total cholesterol, intake of beta-blocker, physical activity, alcohol intake and smoking status. Because of the nonlinear relationship between day time of blood withdrawal and blood glucose a piecewise linear regression was conducted which fitted one slope for morning and one slope for afternoon appointments.

In sensitivity analysis we compared the intraindividual variance of blood glucose by computing age- and sexadjusted differences between (random) first and fasting (second) blood glucose measurement stratified by the time of last caloric intake at the first. The (random) intra-individual variance was assessed by the standard deviation of the test-retest differences $\left(\mathrm{SD}_{\text {diff }}\right)$ of the means (measurement 1 minus measurement 2) with their $95 \%$ confidence intervals (CI) [7]. The $\mathrm{SD}_{\text {diff }}$ can easily be interpreted as $95 \%$ of the random test-rest differences will be less than
$2 * \mathrm{SD}_{\text {diff }}[8]$. All statistical analyses were conducted using SAS 9.2 (SAS Institute, Cary, NC, USA).

\section{Results}

Characteristics of study population and duration of fasting status

Characteristics of the study population stratified by the duration of fasting status are shown in eTable 1. Striking differences according to fasting duration, independent from age, could not be observed. Figure 1 demonstrates a bimodal distribution of fasting duration on the survey day. About $50 \%$ of the participants reported a fasting duration of less then $4 \mathrm{~h}, 28 \%$ more than $\geq 8 \mathrm{~h}$. Only very few participants had a fasting duration of $7 \mathrm{~h}$ or $8 \mathrm{~h}$.

Especially in men the proportion of study participants with a fasting duration $\geq 8$ h decreased with age ( $<36$ years: 34\%, 36-59 years: 30\%, 60-80 years: 25\%, >80 years: $19 \%$; women resp. 26, 23, 21, 20\%).

Blood glucose levels and fasting status

Overall, mean blood glucose levels differed only slightly between fasting and non-fasting individuals: $5.1 \pm 0.8$ $\mathrm{mmol} / \mathrm{L}$ in fasting men, $5.2 \pm 1.2 \mathrm{mmol} / \mathrm{L}$ in non-fasting men; resp. women $4.9 \pm 0.7,5.0 \pm 1.0 \mathrm{mmol} / \mathrm{L}$. Table 1 depicts an age-effect in men, with lowest differences between fasting and non-fasting men in the youngest agegroup $(+0,012 \mathrm{mmol} / \mathrm{L})$ and highest differences in the oldest age-group $\geq 80$ years $(-0.302 \mathrm{mmol} / \mathrm{L})$, reflecting a deteriorating effect of the glucose metabolism with increasing age. This age-effect is not observable in women, but like in men, the oldest women showed the highest blood glucose differences $(-0.032 \mathrm{mmol} / \mathrm{L})$, even though on a much lower mean blood glucose level.

Blood glucose levels and hours of last caloric intake

Figure 2 illustrates the course of age- and sex-adjusted mean blood glucose levels according to hours of last caloric intake. Blood glucose levels of individuals with less than $3 \mathrm{~h}$ of fasting were higher compared to blood glucose levels of individuals with a fasting duration of 8 to more than $18 \mathrm{~h}$ $\left(\mathrm{t}_{\text {Oh }}=5.3 \mathrm{mmol} / \mathrm{L}, \mathrm{t}_{2 \mathrm{~h}}=5.2 \mathrm{mmol} / \mathrm{L}, \mathrm{t}_{8 \mathrm{~h}}=5.0 \mathrm{mmol} / \mathrm{L}\right)$. However, after this time period blood glucose levels are even lower $\left(t_{4 h}, t_{5 h}\right)$ or do not differ anymore $\left(t_{6 h}-t_{7 h}\right)$.

Additionally, stratifying for sex and controlling for time of day did not influence the effect of duration since last caloric intake (eTable 2). Despite the well-known fact of a pronounced age effect on blood glucose levels, the above described time pattern of changes of blood glucose and 


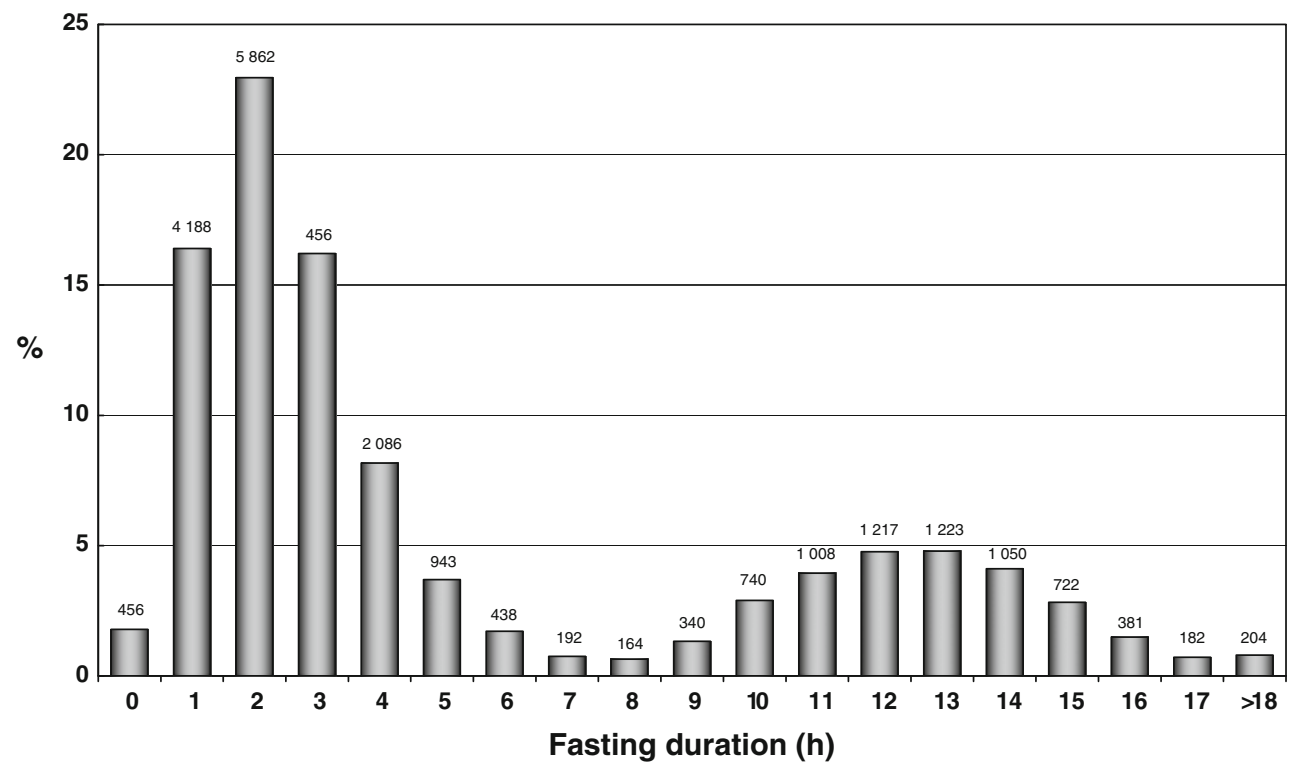

Fig. 1 Distribution of fasting duration on the survey day $(n=25,535)$

Table 1 Difference of mean blood glucose levels (mmol/L) between fasting and non-fasting men and women

\begin{tabular}{|c|c|c|c|c|c|c|}
\hline \multirow{3}{*}{$\begin{array}{l}\text { Age-groups } \\
\text { (years) }\end{array}$} & \multicolumn{4}{|c|}{ Fasting status } & \multirow[t]{3}{*}{ Difference } & \multirow[b]{3}{*}{$P$ value* } \\
\hline & \multicolumn{2}{|c|}{ Fasting ( $\geq 8 \mathrm{~h}$ ) } & \multicolumn{2}{|c|}{ Non-fasting $(<8 \mathrm{~h})$} & & \\
\hline & $\mathrm{n}$ & Mean $\pm \mathrm{SD}$ & $\mathrm{n}$ & Mean $\pm \mathrm{SD}$ & & \\
\hline \multicolumn{7}{|l|}{ Men } \\
\hline$<36$ & 618 & $4.79 \pm 0.61$ & 1,206 & $4.78 \pm 0.86$ & 0.012 & 0.74 \\
\hline $36-59$ & 1,613 & $5.13 \pm 0.86$ & 3,785 & $5.21 \pm 1.19$ & -0.082 & 0.0045 \\
\hline $60-80$ & 759 & $5.37 \pm 0.84$ & 2,242 & $5.50 \pm 1.34$ & -0.128 & 0.0022 \\
\hline$\geq 80$ & 38 & $5.43 \pm 0.65$ & 167 & $5.73 \pm 1.46$ & -0.302 & 0.0529 \\
\hline All & 3,028 & $5.13 \pm 0.83$ & 7,400 & $5.24 \pm 1.22$ & -0.115 & $<0.001$ \\
\hline \multicolumn{7}{|l|}{ Women } \\
\hline$<36$ & 924 & $4.57 \pm 0.49$ & 2,602 & $4.60 \pm 0.69$ & -0.032 & 0.1272 \\
\hline $36-59$ & 2,196 & $4.91 \pm 0.63$ & 7,356 & $4.95 \pm 0.92$ & -0.037 & 0.0303 \\
\hline $60-80$ & 868 & $5.21 \pm 0.81$ & 3,260 & $5.23 \pm 1.09$ & -0.018 & 0.5864 \\
\hline$\geq 80$ & 79 & $5.27 \pm 0.74$ & 311 & $5.43 \pm 1.14$ & -0.162 & 0.1261 \\
\hline All & 4,067 & $4.90 \pm 0.69$ & 13,529 & $5.0 \pm 0.96$ & -0.056 & $<0.001$ \\
\hline
\end{tabular}

Fasting: last caloric intake $\geq 8 \mathrm{~h}$, non-fasting: last caloric intake $<8 \mathrm{~h}$

* $P$ values refer to $t$-tests differences between fasting and nonfasting blood glucose in each age-and sex-specific group

${ }^{\mathrm{a}}$ Difference $=($ last caloric intake $\geq 8 \mathrm{~h})-($ last caloric intake $<8 \mathrm{~h})$

fasting duration is similar to all but very old participants $>80$ years (Fig. 3 ).

Control of external influence factors

Table 2 shows the sex-specific impact of fasting duration on blood glucose levels additionally adjusted for waist circumference, metabolic and lifestyle factors. With each additional hour of fasting the blood glucose decreases by about $0.024 \mathrm{mmol} / \mathrm{L}(\stackrel{\triangleq}{\underline{0}} 0.4 \mathrm{mg} / \mathrm{dL})$ in men and $0.009 \mathrm{mmol} / \mathrm{L}$ $(\bumpeq 0.2 \mathrm{mg} / \mathrm{dL})$ in women. Theoretically, adding this up to $8 \mathrm{~h}$ (irrespective of day of blood sampling) this amounts to about $3 \mathrm{mg} / \mathrm{dL}$ in men and about $2 \mathrm{mg} / \mathrm{dL}$ in women, confirming the above reported results of marginal higher blood glucose levels comparing fasting and non-fasting blood glucose concentrations. In addition to the well known associations of blood glucose with CVD risk factors (lipids, blood pressure, obesity), we observed a strong association with time of day of blood sampling (Table 2). Notably this association was only observable when stratifying our analysis by sex, 


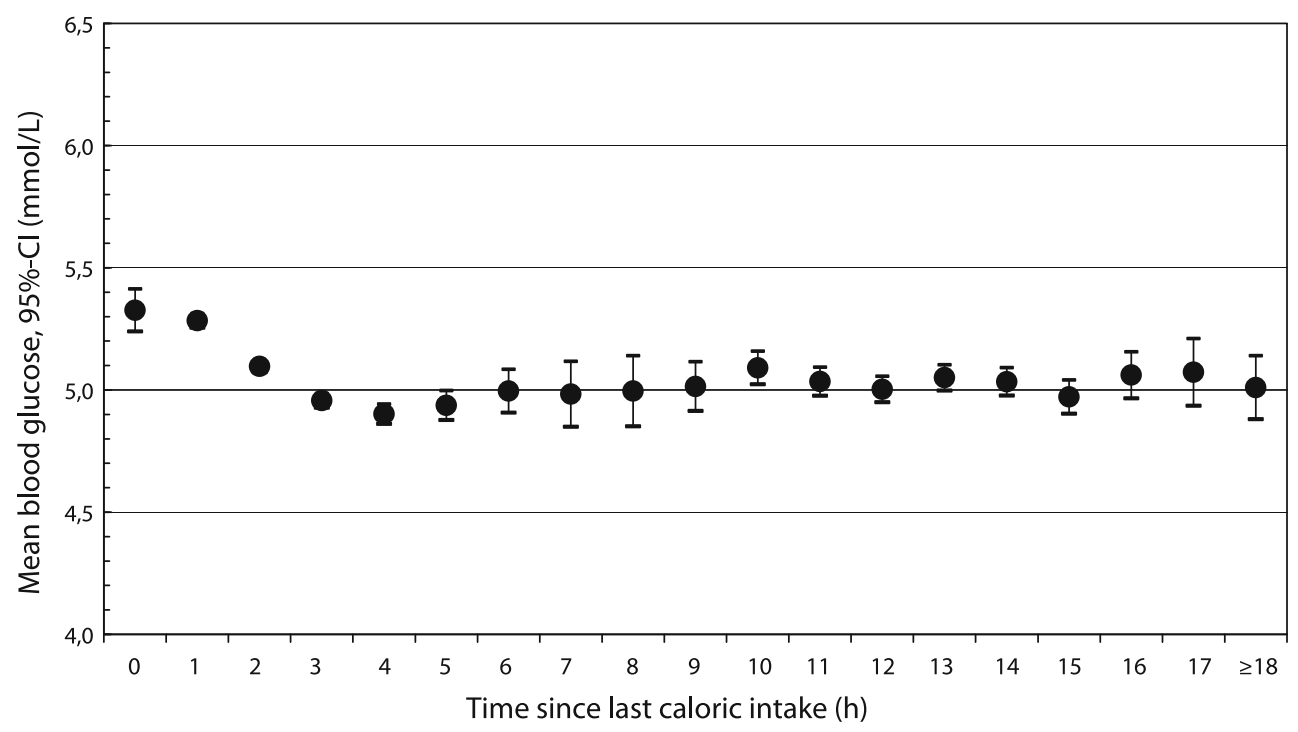

Fig. 2 Age- and sex-adjusted blood glucose levels by time since last caloric intake $(\mathrm{n}=25,535)$

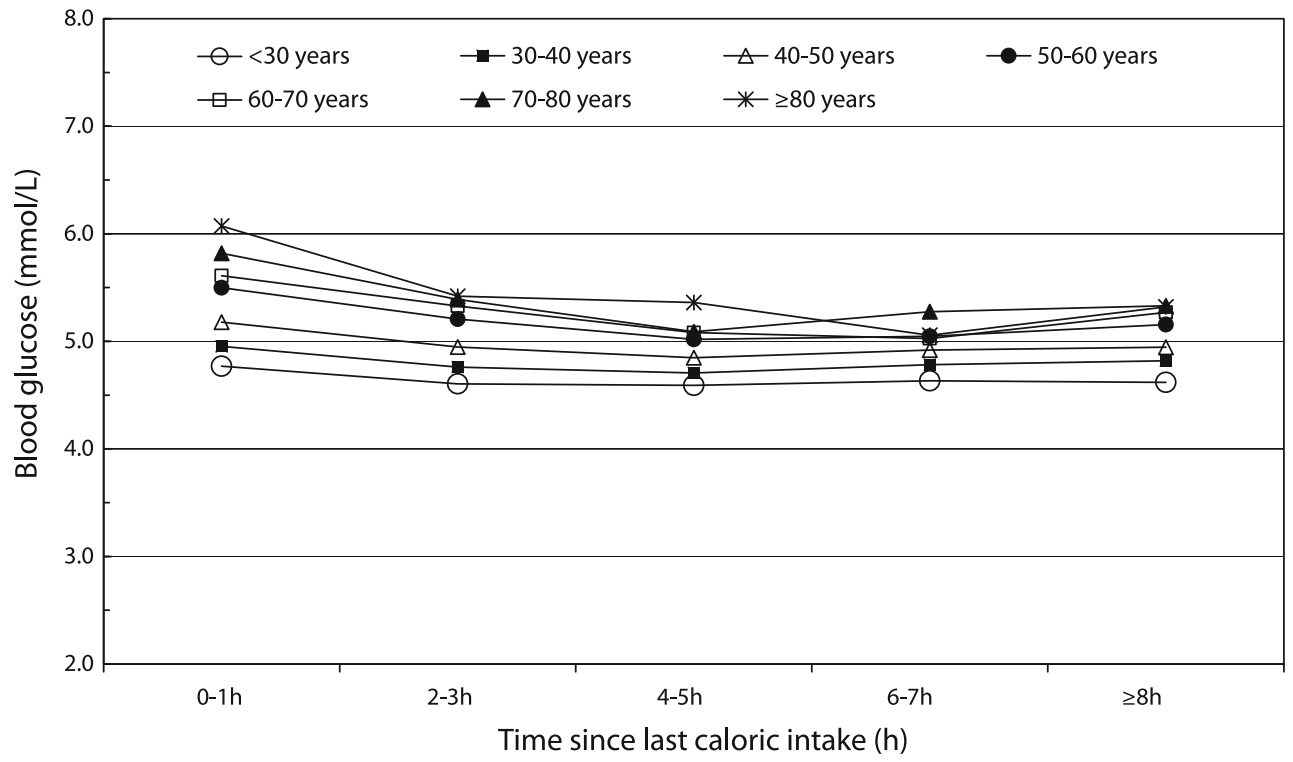

Fig. 3 Sex-adjusted blood glucose levels by time since last caloric intake stratified by age-groups

demonstrating a bidirectional trend between sampling in the morning and in the afternoon.

Sensitivity analyses

As expected, the intra-individual variability differed with regard to fasting status on two consecutive measurements. The spearman correlation coefficient was highest with a fasting status at both measurements, reaching 0.625 (eFigure 1). The coefficient diminished to 0.388 comparing a first random versus a second fasting measurement, which did not differ from two consecutive measurements both at random (0.386).
However, the mean age- and sex-adjusted difference between a first (random) and a second (fasting) blood glucose measurement, stratified by fasting duration at the first measurement (eFigure 2), shows the highest difference $(+0.66 \mathrm{mmol} / \mathrm{L})$ when fasting status is less than $1 \mathrm{~h}$ compared to the fasting blood glucose at the second measurement and no differences when fasting was $\geq 4 \mathrm{~h}$, confirming the previous results. Restriction of this analysis to the random sample of participants did not change our results substantially.

Differences in blood glucose between first and second measurement were remarkable small regardless of fasting status of first and second measurement (fasting/fasting, 
Table 2 Association between blood glucose and fasting duration, time of blood sampling and personal characteristics stratified by sex

\begin{tabular}{|c|c|c|c|c|}
\hline & \multicolumn{2}{|l|}{ Men } & \multicolumn{2}{|l|}{ Women } \\
\hline & \multicolumn{2}{|c|}{ Change of blood glucose (mmol/L) } & \multicolumn{2}{|c|}{ Change of blood glucose (mmol/L) } \\
\hline & B & $95 \%-\mathrm{CI}$ & ß & $95 \%-\mathrm{CI}$ \\
\hline Age (years) & 0.010 & $0.008 ; 0.011$ & 0.010 & $0.009 ; 0.012$ \\
\hline Fasting duration (h) & -0.024 & $-0.029 ;-0.018$ & -0.009 & $-0.013 ;-0.006$ \\
\hline \multicolumn{5}{|l|}{ Time of day of blood sampling } \\
\hline Morning ( < 12.00 p.m.) & -0.052 & $-0.075 ;-0.029$ & -0.027 & $-0.042 ;-0.011$ \\
\hline Afternoon ( $\geq 12.00$ p.m.) & 0.011 & $-0.015 ; 0.037$ & 0.020 & $0.005 ; 0.035$ \\
\hline HDL-cholesterol (mmol/L) & -0.120 & $-0.189 ;-0.050$ & -0.095 & $-0.132 ;-0.058$ \\
\hline Total cholesterol (mmol/L) & -0.003 & $-0.026 ; 0.021$ & 0.018 & $0.001 ; 0.034$ \\
\hline Systole blood pressure (mmHg) & 0.004 & $0.002 ; 0.005$ & 0.002 & $0.002 ; 0.003$ \\
\hline Intake of beta-blocker & 0.160 & $0.100 ; 0.220$ & 0.152 & $0.111 ; 0.193$ \\
\hline Waist circumference $(\mathrm{cm})$ & 0.010 & $0.008 ; 0.012$ & 0.008 & $0.006 ; 0.009$ \\
\hline Smoking & 0.048 & $-0.004 ; 0.100$ & 0.022 & $-0.013 ; 0.056$ \\
\hline \multicolumn{5}{|l|}{ Beverage } \\
\hline Soft/fizzy drinks ${ }^{\mathrm{a}}$ & 0.004 & $-0.054 ; 0.062$ & 0.046 & $0.001 ; 0.089$ \\
\hline Hard liquor $^{\mathrm{a}}$ & 0.234 & $0.053 ; 0.414$ & 0.212 & $0.014 ; 0.409$ \\
\hline Physical activity (ref. $<2$ h/week) & -0.096 & $-0.150 ;-0.042$ & -0.034 & $-0.069 ; 0.001$ \\
\hline
\end{tabular}

${ }^{a}$ Reference for beverages $=$ never and seldom

Table 3 Sex-specific intraindividual variability in blood glucose measured by two consecutive appointments according to fasting status

\begin{tabular}{lrrrr}
\hline & \multicolumn{4}{c}{$\Delta_{\text {measurement } 1-\text { measurement } 2(\mathrm{mmol} / \mathrm{L})}$} \\
\cline { 3 - 5 } & $\mathrm{n}$ & $\mathrm{Mean}_{\mathrm{dif}}$ & \multicolumn{1}{c}{$95 \%$-CI } & $\mathrm{SD}_{\mathrm{dif}}$ \\
\hline Men & 612 & 0.047 & $-0.004 ; 0.099$ & 0.653 \\
Fasting-fasting & 64 & -0.053 & $-0.274 ; 0.168$ & 0.886 \\
Non fasting-fasting & 3,099 & 0.344 & $0.301 ; 0.388$ & 1.234 \\
Fasting-nonfasting & 425 & 0.024 & $-0.102 ; 0.150$ & 1.320 \\
Nonfasting-nonfasting & & & & \\
Women & 664 & 0.112 & $0.051 ; 0.172$ & 0.794 \\
Fasting-fasting & 94,472 & 0.275 & $0.247 ; 0.304$ & 0.975 \\
Non fasting-fasting & -0.130 & $-0.313 ; 0.053$ & 0.892 \\
Fasting-nonfasting & 729 & 0.153 & $0.072 ; 0.233$ & 1.108 \\
Nonfasting-nonfasting & 729 &
\end{tabular}

$S D_{\text {dif }}$ standard deviation of the first and second measurement differences

Fasting $\geq 8 \mathrm{~h}$, non-fasting $<8 \mathrm{~h}$ since last caloric intake, $95 \%$-CI: $95 \%$ confidence interval

non-fasting/fasting, etc.), despite a lower varianceexpressed as the standard deviation of the mean difference -in the fasting/fasting combination (Table 3).

At least we tested intra-individual changes of assignment to blood glucose-risk categories (low: $<5.6 \mathrm{mmol} / \mathrm{L}$, medium: $5.6-7.0 \mathrm{mmol} / \mathrm{L}$, high $\geq 7.0 \mathrm{mmol} / \mathrm{L}$ ) at the repeated second fasting measurement compared to different fasting durations $(\geq 8,>0, \geq 3 \mathrm{~h})$ at first measurement. We found that $91.8 \%$ of those individuals with a low fasting blood glucose $(\geq 8 \mathrm{~h})$ at first measurement remained in the low category at the repeated measurement (Table 4). This proportion attenuated slightly comparing those with a random fasting $(>0 \mathrm{~h})$ and a fasting status $\geq 3 \mathrm{~h}$ at first measurement $(90.6 \%$, resp. $89.3 \%)$. On the other hand, in only $22.2 \%$ of the individuals with a fasting duration $\geq 8 \mathrm{~h}$, resp. $18.4 \%$ with a fasting duration $\geq 3 \mathrm{~h}$ and $11.3 \%$ with a random measurement, a high blood glucose level could be confirmed at the second measurement. Hence, transitions between the medium and high categories were highindependent of fasting duration at first and second measurement.

\section{Discussion}

In our study population of women and men, aged 1899 years, the minimal fasting duration to achieve comparable blood glucose levels between fasting and non-fasting measurements was $3 \mathrm{~h}$. Overall, mean blood glucose levels differed only marginally between fasting and non-fasting individuals. Although the variance of blood glucose was higher in men and older individuals, the results are independently from age and sex. This pattern persisted even when taking into account varying waist circumference, metabolic and lifestyle factors. Analyzing intraindividual changes between non-fasting and fasting measurements confirmed these results, underlining the robustness of our findings. Moreover, our data show strong associations between blood glucose levels and time of day of blood 
Table 4 Intraindividual change of blood glucose categories at second (fasting) measurement compared to a fasting duration of $\geq 8 \mathrm{~h}$, $>0 \mathrm{~h}$ (random) and $\geq 3 \mathrm{~h}$ at first measurement

\begin{tabular}{|c|c|c|c|c|c|c|c|c|c|}
\hline \multicolumn{2}{|l|}{ 1. Measurement } & \multicolumn{8}{|c|}{ 2. Fasting measurement $\geq 8 \mathrm{~h}$} \\
\hline \multirow[t]{2}{*}{ Fasting duration (h) } & \multirow[t]{2}{*}{ Blood glucose category ${ }^{a}$} & \multicolumn{2}{|l|}{ Low } & \multicolumn{2}{|c|}{ Medium } & \multicolumn{2}{|l|}{ High } & \multicolumn{2}{|l|}{ All } \\
\hline & & $\%$ & $\mathrm{n}$ & $\%$ & $\mathrm{n}$ & $\%$ & $\mathrm{n}$ & $\%$ & $\mathrm{n}$ \\
\hline$\geq 8$ & Low & 91.8 & 938 & 7.9 & 81 & 0.3 & 3 & 80.2 & 1,022 \\
\hline$>0$ (random) & & 90.6 & 4,966 & 8.7 & 479 & 0.7 & 37 & 72.5 & 5,482 \\
\hline$\geq 3$ & & 89.3 & 2,473 & 10.3 & 278 & 0.7 & 20 & 79.6 & 2,771 \\
\hline$\geq 8$ & Medium & 48.2 & 109 & 45.6 & 103 & 6.2 & 14 & 17.7 & 226 \\
\hline$>0$ (random) & & 77.0 & 1,220 & 21.6 & 343 & 1.4 & 22 & 21.0 & 1,585 \\
\hline$\geq 3$ & & 67.0 & 382 & 30.9 & 176 & 2.1 & 12 & 21.0 & 1,101 \\
\hline$\geq 8$ & High & 40.7 & 11 & 37.0 & 10 & 22.2 & 6 & 2.1 & 27 \\
\hline$>0$ (random) & & 57.5 & 284 & 31.2 & 154 & 11.3 & 56 & 6.5 & 494 \\
\hline$\geq 3$ & & 48.9 & 69 & 32.6 & 46 & 18.4 & 26 & 4.1 & 141 \\
\hline$\geq 8$ & All & 83.0 & 1,085 & 15.2 & 194 & 1.8 & 23 & 100 & 1,275 \\
\hline$>0$ (random) & & 85.6 & 6,470 & 12.9 & 976 & 1.5 & 115 & 100 & 7,561 \\
\hline$\geq 3$ & & 84.0 & 2,924 & 14.4 & 500 & 1.7 & 58 & 100 & 3,482 \\
\hline
\end{tabular}

${ }^{a}$ Categories defined as a low blood glucose $<5.6 \mathrm{mmol} / \mathrm{L}$, medium blood glucose 5.6-7.0 mmol/L, high blood glucose $\geq 7.1 \mathrm{mmol} / \mathrm{L}$

withdrawal, regular physical activity or drinking of hard liquor.

Fasting versus non-fasting blood glucose measurements

To the best of our knowledge, evidence is missing demonstrating that blood glucose measurements with a fasting duration of more than $8 \mathrm{~h}$ do better than those with less than $8 \mathrm{~h}$ of fasting duration. The main argument for fasting glucose measurements is the rise in blood glucose levels seen after a caloric meal or drink, especially during an oral glucose tolerance test (oGTT) [9]. However, our data show higher mean blood glucose levels only in the first $3 \mathrm{~h}$ after a caloric intake. This observation is in line with an earlier study from the fifties [10] and the ADA in 2001, stating that in nondiabetic individuals, blood glucose levels return to preprandial levels within 2-3 h [11]. In the British Regional Heart Study, a cross-sectional study of 60-79 year old men [12], no difference of blood glucose levels between fasting $<6 \mathrm{~h}$ versus $\geq 6 \mathrm{~h}$ were found $(5.6 \mathrm{mmol} / \mathrm{L}$ resp. $5.7 \mathrm{mmol} /$ $\mathrm{L}$ ), which is in accordance to our results (Table 1). Further studies - mainly focussing on the effect of diurnal variations of blood glucose levels or postprandial glucose metabolism -report a decrease of blood glucose to normal blood glucose levels 2-3 h after an oral glucose tolerance test or test meal [9, 13-15], supporting our findings of small differences between fasting and non-fasting glucose levels.

To maintain glucose homeostasis, the human body is able to tightly regulate levels of circulating blood glucose by coupling nutrient-stimulated insulin secretion (insulin response) and the metabolic action of insulin to stimulate glucose disposal [16]. In individuals with diabetes the glucose homeostatis is disturbed. Diabetics not only exceed normal blood glucose levels, but also experience high blood glucose fluctuations, with a higher increase of blood glucose after caloric intake and a prolonged decrease to baseline levels compared to normal individuals. Thus we excluded participants with known diabetes mellitus from our analysis.

Increasing age is associated with increasing impairment of the glucose homeostasis, possibly due to a decreasing insulin secretory capacity or insulin action [17]. Our data show higher blood glucose differences between fasting and non-fasting participants in the oldest age-group, especially in men (Table 1). However, these differences are rather low and they seem to be no reason for a fasting duration of more than $3 \mathrm{~h}$.

Blood glucose levels are influenced by analytical conditions [18] and (intra-individual) biologic variations [1922]. The latter is dependent on a number of factors like time of day, medication intake prior exercise status or alcohol intake [22-25]. The observed maximum age- and sex-adjusted blood glucose increase in our study of $0.3 \mathrm{mmol} / \mathrm{L}(\triangleq 5.4 \mathrm{mg} / \mathrm{dL})$ with regard to duration of last caloric intake is very like within the variation of these biologic factors.

Strengths and limitations

One strength of our study is the large sample size and the broad age-range, allowing us to study the influence of age 
and sex in detail. A notable gain of our study is the repeated blood glucose measurement in a subsample of participants. Thus, we were able to determine in sensitivity analyses directly the intra-individual variability with respect to fasting status. Since time of last caloric intake was self-reported and therefore subject to error, participants not only were asked by the doctor for the exact time of last caloric intake, but additionally for the type of last meal and drink. Accordingly, participants more likely remember the right time, which in turn should reduce this potential recall bias. In any case, other methods to measure last caloric intake with less bias are apparently unacceptable for study participants or patients. Our participants were mostly white and recruited from different geographically located general practices across Germany. Thus our results may not apply to other ethnic groups. A detailed discussion of possible selection bias of our study is provided in [5]. Briefly, characteristics of this primary health care sample are comparable to other German populationbased samples and to the German federal statistical data with regard to anthropometric measures, smoking status, marital status, schooling and unemployment rate (i.e. GEMCAS: $10.2 \%$, Germany October 2005: 10.4\%). This high conformance might be explained by the situation that $92 \%$ of adults in Germany consult a general practitioner during 1 year [26]. However, the proportion of participants with diabetes and CVD is higher than compared to population-based samples, but still lower than in real patientbased samples [5]. We found differences in demographic characteristics in fasting versus non-fasting individuals. To account for these differences we stratified by sex and agegroups and estimated adjusted associations; nonetheless residual confounding in this observational study might still be an issue. Overall, our results have to be interpreted in terms of the observational nature of our study.

\section{Implications for blood sampling in clinical practice and research}

Possible implications for blood sampling regimes depend on the intention of blood glucose measurements. In clinical practice blood glucose is basically measured in order to obtain information about the existence and extent (control and treatment of diabetes) of a disturbed glucose metabolism (diagnosis of diabetes mellitus). According to recommendations of international experts a diagnosis of diabetes mellitus is possible in different ways: random or fasting blood glucose measurement or oGTT. For the ADA a confirmation of the test on a subsequent day is mandatory in the absence of unequivocal hyperglycemia [27]. In this sense the recommendation of the WHO is even stricter stating “... the diagnosis of diabetes in an asymptomatic subject should never be made on the basis of a single abnormal blood glucose value. For the asymptomatic person, at least one additional plasma/blood glucose test result with a value in the diabetic range is essential, either fasting, from a random (casual) sample, or from the OGTT" [1]. The importance of a replicate test is supported by our data, showing high transition rates between medium and high blood glucose categories - independent of fasting duration. Thus, several methods for a diagnosis of diabetes are already available, allowing clinicians to apply the most advantageous method in their everyday practice and the most convenient method for their patients.

One argument in favour of recommending a fasting measurement might be the often concurrent determination of lipid profiles for CVD risk assessment. However, according to recent studies [28-32] and recommendations [33-36] fasting blood glucose measurements for clinical purposes are not necessary in the first place, even in combination with the determination of lipid profiles for CDV risk assessment.

Implications for research purposes depend on the study question to be studied. (1) Aetiological studies aiming to understand glucose metabolism or the development of diabetes mellitus certainly need highly standardized conditions concerning fasting status. Anyhow, our results confirm that - beyond the fasting status - a thorough control of a wide range of factors is necessary to achieve unbiased measurements. (2) In epidemiological studies blood glucose is often measured as risk factor, mediator or confounder. In this case, our results not only challenge the current need of a fasting duration of more than $8 \mathrm{~h}$, but also suggest a random sampling accomplished by a detailed assessment of last caloric intake or - if feasible - a fasting duration of $3 \mathrm{~h}$ as sufficient. As discussed above, fasting measurements are not necessarily unbiased, as marked variations due to a wide range of external and internal influencing factors still occur.

In conclusion, our data challenge the necessity for a fasting duration of $>8 \mathrm{~h}$ when measuring blood glucose. A random blood glucose sample or a fasting duration of $3 \mathrm{~h}$ seems sufficient for reliable blood glucose measurements. Rather, our study indicates that essentially more effort on the assessment of additional external/internal factors on blood glucose levels is necessary. Blood glucose assessment should be accomplished by a detailed assessment of last caloric meal and drink as well as a thorough assessment of alcohol consumption, medication intake, exercise status, and time of day of blood sampling. Further studies are warranted, systematically studying the effect of different fasting duration on blood glucose levels. In case of a confirmation of our results, this would simplify routines in clinical practices for both the clinicians and the patients and would facilitate the scheduling appointments at epidemiological studies with a considerable cost reducing effect. 
Acknowledgments We would like to thank the physicians and their personnel involved in the study for their contribution. Also, we are indebted to all study participants for their kind cooperation in examinations and interviews. We are very grateful to Barbara Hoffmann and Chakrapani Balijepalli for their carefully reading of the manuscript and their helpful scientific commentaries improving our manuscript. This work was supported by an unrestricted educational research grant by Sanofi Aventis Deutschland GmbH, Berlin, Germany.

Conflict of interest None declared.

Open Access This article is distributed under the terms of the Creative Commons Attribution Noncommercial License which permits any noncommercial use, distribution, and reproduction in any medium, provided the original author(s) and source are credited.

\section{References}

1. World Health Organization. Definition and diagnosis and classification of diabetes mellitus and its complications: report of a WHO consultation. Part 1. Geneva, Switzerland: WHO; 1999. pp. 32-33.

2. The Expert Committee on the diagnosis and classification of diabetes mellitus. Report of the expert committee on the diagnosis and classification of diabetes mellitus. Diabetes Care 1997; 20:1183-1197.

3. American Diabetes Association. Diagnosis and classification of diabetes mellitus. Diabetes Care. 2010;33(Suppl 1):S62-9.

4. Moebus S, Hanisch J, Aidelsburger P, Bramlage P, Wasem J, Jöckel KH. Impact of 4 different definitions used for the assessment of the prevalence of the metabolic syndrome in primary healthcare: the german metabolic and cardiovascular risk project (GEMCAS). Cardiovasc Diabetol. 2007;6(1):22.

5. Moebus S, Hanisch J, Neuhaeuser M, Aidelsburger P, Wasem J, Joeckel K-H. Assessing the prevalence of the metabolic syndrome according to NCEP ATP III in Germany: feasibility and quality aspects of a two step approach in 1550 randomly selected primary health care practices. GMS Ger Med Sci 2006; 4:Doc07, http://www.egms.de/en/gms/2006-4/000036.shtml.

6. Deutsche Arbeitsgemeinschaft für Epidemiologie. Leitlinien und Empfehlungen zur Sicherung von Guter Epidemiologischer Praxis (GEP). 2004. http://www.gesundheitsforschung-bmbf.de/_ media/Empfehlungen_GEP.pdf.

7. Martin Bland J, Altman D. Statistical methods for assessing agreement between two methods of clinical measurement. Lancet. 1986;327(8476):307-10.

8. Mooy JM, Grootenhuis GH, Vries $\mathrm{H}$, et al. Intra-individual variation of glucose, specific insulin and proinsulin concentrations measured by two oral glucose tolerance tests in a general Caucasian population: the Hoorn study. Diabetologia. 1996;39(3):298305.

9. Ahmed M, Gannon MC, Nuttall FQ. Postprandial plasma glucose, insulin, glucagon and triglyceride responses to a standard diet in normal subjects. Diabetologia. 1976;12(1):61-7.

10. Sindoni A. Fasting blood sugar vs. postprandial blood sugar as observed in normal indiviudals, medical (non-diabetic) patients, and patients with diabetes. Am J Dig Dis. 1946;13(6):178-92.

11. American Diabetes Association. Postprandial blood glucose. Diabetes Care. 2001;24(4):775-8.

12. Emberson JR, Whincup PH, Walker M, Thomas M, Alberti KGMM. Biochemical measures in a population-based study: effect of fasting duration and time of day. Ann Clin Biochem. 2002;39(5):493-501.
13. Pocock SJ, Ashby D, Shaper AG, Walker M, Broughton PM. Diurnal variations in serum biochemical and haematological measurements. J Clin Pathol. 1989;42(2):172-9.

14. Troisi RJ, Cowie CC, Harris MI. Diurnal variation in fasting plasma glucose: implications for diagnosis of diabetes in patients examined in the afternoon. JAMA. 2000;284(24):3157-9.

15. Meier JJ, Baller B, Menge BA, Gallwitz B, Schmidt WE, Nauck MA. Excess glycaemic excursions after an oral glucose tolerance test compared with a mixed meal challenge and self-measured home glucose profiles: is the OGTT a valid predictor of postprandial hyperglycaemia and vice versa? Diabetes Obes Metab. 2008;11(3):213-22.

16. Stumvoll M, Tataranni PA, Stefan N, Vozarova B, Bogardus C. Glucose allostasis. Diabetes. 2003;52(4):903-9.

17. Basu R, Man CD, Campioni M, Basu A, Klee G, Toffolo G, Cobelli C, Rizza RA. Effects of age and sex on postprandial glucose metabolism: differences in glucose turnover, insulin secretion, insulin action, and hepatic insulin extraction. Diabetes. 2006;55:2001-14.

18. Young DS, Harris EK, Cotlove E. Biological and analytic components of variation in long-term studies of serum constituents in normal subjects: IV. Results of a study designed to eliminate long-term analytic deviations. Clin Chem. 1971;17(5):403-10.

19. Harris EK, Kanofsky P, Shakarji G, Cotlove E. Biological and analytic components of variation in long-term studies of serum constituents in normal subjects: II. Estimating biological components of variation. Clin Chem. 1970;16(12):1022-7.

20. Williams GZ, Widdowson GM, Penton J. Individual character of variation in time-series studies of healthy people: II. Differences in values for clinical chemical analytes in serum among demographic groups, by age and sex. Clin Chem. 1978;24(2):313-20.

21. Godsland IF. Intra-individual variation: significant changes in parameters of lipid and carbohydrate metabolism in the individual and intra-individual variation in different test populations. Ann Clin Biochem. 1985;22:618-24.

22. NIH Consensus conference. Triglyceride, high-density lipoprotein, and coronary heart disease. NIH consensus development panel on triglyceride, high-density lipoprotein, and coronary heart disease. JAMA. 1993;269(4):505-10.

23. Van Cauter E, Polonsky KS, Scheen AJ. Roles of circadian rhythmicity and sleep in human glucose regulation. Endocr Rev. 1997;18(5):716-38.

24. Goodyear LJ, Kahn BB. Exercise, glucose transport, and insulin sensitivity. Ann Rev Med. 1998;49:235-61.

25. Mumford SL, Schisterman EF, Siega-Riz AM et al. A longitudinal study of serum lipoproteins in relation to endogenous reproductive hormones during the menstrual cycle: findings from the biocycle study. J Clin Endocrinol Metab. 2010;95(9):E80E85.

26. Pittrow D, Bramlage P, Hoefler $M$, et al. Praevalenz und Schweregrad von arterieller Hypertonie und Diabetes mellitus in der hausaerztlichen Praxis-ein unterschätztes Problem. J für Hypertonie. 2003;7(4):7-11.

27. American Diabetes Association. Diagnosis and classification of diabetes mellitus. Diabetes Care. 2004;27(suppl 1):s5-10.

28. Schaefer EJ, Audelin MC, McNamara JR, et al. Comparison of fasting and postprandial plasma lipoproteins in subjects with and without coronary heart disease. Am J Cardiol. 2001;88(10):1129_ 1133.

29. Langsted A, Freiberg JJ, Nordestgaard BG. Fasting and nonfasting lipid levels: influence of normal food intake on lipids, lipoproteins, apolipoproteins, and cardiovascular risk prediction. Circulation. 2008;118(20):2047-56.

30. Mora S, Rifai N, Buring JE, Ridker PM. Fasting compared with nonfasting lipids and apolipoproteins for predicting incident cardiovascular events. Circulation. 2008;118(10):993-1001. 
31. Bansal S, Buring JE, Rifai N, Mora S, Sacks FM, Ridker PM. Fasting compared with nonfasting triglycerides and risk of cardiovascular events in women. JAMA. 2007;298(3):309-16.

32. Freiberg JJ, Tybjaerg-Hansen A, Jensen JS, Nordestgaard BG. Nonfasting triglycerides and risk of ischemic stroke in the general population. JAMA. 2008;300(18):2142-52.

33. Executive summary of the third report of the National Cholesterol Education Program (NCEP) expert panel on detection, evaluation, and treatment of high blood cholesterol in adults (adult treatment panel III). JAMA 2001; 285(19):2486-2497.

34. De Backer G, Ambrosioni E, Borch-Johnsen K, et al. European guidelines on cardiovascular disease prevention in clinical practice. Third joint task force of European and other societies on cardiovascular disease prevention in clinical practice. Eur Heart J. 2003;24(17):1601-10.

35. British Cardiac Society, British Hypertension Society, Diabetes UK, HEART UK, Primary Care Cardiovascular Society, Stroke Association. JBS 2: Joint British Societies' guidelines on prevention of cardiovascular disease in clinical practice. Heart 2005; 91(Suppl 5), v1-v52.

36. Nordestgaard BG, Benn M. Fasting and nonfasting LDL cholesterol: to measure or calculate? Clin Chem. 2009;55(5):845-7.

37. Grundy SM, Cleeman JL, Daniels SR, Donato KA, Eckel RH, Franklin BA, et al. Diagnosis and management of the metabolic syndrome: an American Heart Association/National Heart, Lung, and blood Institute scientific statement. Circulation. 2005;112:2735-52. 